\title{
New Drug Approvals
}

The following drugs have recently been approved by the FDA. These include newly approved drugs and new indications.

\section{Quviviq (daridorexant) Tablets}

Date of Approval: January 7, 2022

Company: Idorsia Ltd.

Treatment for: Insomnia

Quviviq (daridorexant) is a dual orexin receptor antagonist (DORA) for the treatment of insomnia.

\section{Xaciato (clindamycin phosphate) Vaginal Gel- formerly DARE-BV1}

Date of Approval: December 7, 2021

Company: Daré Bioscience, Inc.

Treatment for: Bacterial Vaginosis

Xaciato (clindamycin phosphate) is a topical lincosamide antibacterial for the vaginallyadministered treatment of bacterial vaginosis in female patients of 12 years of age and older.

\section{Entadfi (finasteride and tadalafil) Capsules}

Date of Approval: December 9, 2021

Company: Veru Inc.

Treatment for: Benign Prostatic Hyperplasia

Entadfi (finasteride and tadalafil) is a $5 \alpha$-reductase inhibitor and phosphodiesterase 5 (PDE5) inhibitor combination indicated for the treatment of urinary tract symptoms caused by benign prostatic hyperplasia (BPH).

Tarpeyo (budesonide) Delayed Release Capsules formerly Nefecon

Date of Approval: December 15, 2021

Company: Calliditas Therapeutics $\mathrm{AB}$

Treatment for: IgA Nephropathy

Tarpeyo (budesonide) is a targeted release formulation of the approved corticosteroid budesonide indicated to reduce proteinuria in adults with primary immunoglobulin A nephropathy (IgAN).

\section{Dartisla ODT (glycopyrrolate) Orally Disintegrating Tablets}

Date of Approval: December 16, 2021

Company: Edenbridge Pharmaceuticals, LLC

Treatment for: Peptic Ulcer

Dartisla ODT (glycopyrrolate) is an anticholinergic indicated in adults to reduce symptoms of a peptic ulcer as an adjunct to treatment of peptic ulcer.

\section{Tezspire (tezepelumab-ekko) Injection}

Date of Approval: December 17, 2021

Company: Amgen and AstraZeneca

Treatment for: Asthma

Tezspire (tezepelumab-ekko) is a thymic stromal lymphopoietin (TSLP) blocker, human monoclonal antibody (IgG2 $\lambda$ ), indicated for the add-on maintenance treatment of adult and pediatric patients aged 12 years and older with severe asthma.

\section{Vyvgart (efgartigimod alfa-fcab) Injection}

Date of Approval: December 17, 2021

Company: Argenx SE

Treatment for: Myasthenia Gravis

Vyvgart (efgartigimod alfa-fcab) is a neonatal Fc receptor blocker indicated for the treatment of generalized myasthenia gravis (gMG) in adult patients who are anti-acetylcholine receptor (AChR) antibody positive.

\section{Yusimry (adalimumab-aqvh) Injection}

Date of Approval: December 17, 2021

Company: Coherus BioSciences, Inc.

Treatment for: Rheumatoid Arthritis, Juvenile

Rheumatoid Arthritis, Psoriatic Arthritis, Ankylosing Spondylitis, Crohn's Disease, Ulcerative Colitis, Plaque Psoriasis

Yusimry (adalimumab-aqvh) is a tumor necrosis factor (TNF) blocker biosimilar to Humira, approved for the treatment of rheumatoid arthritis, juvenile idiopathic arthritis, psoriatic arthritis, ankylosing spondylitis, Crohn's disease, ulcerative colitis, and plaque psoriasis. 


\section{Apretude (cabotegravir) Extended-Release Injectable Suspension}

Date of Approval: December 20, 2021

Company: ViiV Healthcare

Treatment for: Pre-Exposure Prophylaxis

Apretude (cabotegravir) is a long-acting injectable integrase strand transfer inhibitor (INSTI) indicated for pre-exposure prophylaxis (PrEP) to reduce the risk of sexually acquired HIV-1 infection.

\section{Leqvio (inclisiran) Injection}

Date of Approval: December 22, 2021

Company: Novartis

Treatment for: High Cholesterol, Familial

Heterozygous

Leqvio (inclisiran) is a small interfering RNA (siRNA) directed to PCSK9 (proprotein convertase subtilisin kexin type 9) mRNA used to reduce lowdensity lipoprotein cholesterol.

\section{Adbry (tralokinumab-ldrm) Injection}

Date of Approval: December 27, 2021

Company: LEO Pharma Inc.

Treatment for: Atopic Dermatitis

Adbry (tralokinumab-ldrm) is an interleukin-13 antagonist used for the treatment of moderate-tosevere atopic dermatitis.

\section{Recorlev (levoketoconazole) Capsules}

Date of Approval: December 30, 2021

Company: Xeris Biopharma Holdings

Treatment for: Cushing's Syndrome

Recorlev (levoketoconazole) is a cortisol synthesis inhibitor indicated for the treatment of endogenous hypercortisolemia in adult patients with Cushing's syndrome.

\section{Eprontia (topiramate) Oral Solution}

Date of Approval: November 5, 2021

Company: Azurity Pharmaceuticals, Inc.

Treatment for: Epilepsy, Migraine Prevention

Eprontia (topiramate) is a ready-to-use liquid formulation of the approved anticonvulsant topiramate for use in the treatment of epilepsy and the preventive treatment of migraine.
Besremi (ropeginterferon alfa-2b-njft) Injection

Date of Approval: November 12, 2021

Company: PharmaEssentia Corporation

Treatment for: Polycythemia Vera

Besremi (ropeginterferon-alfa-2b-njft) is an interferon alfa- $2 b$ indicated for the treatment of adults with polycythemia vera.

Voxzogo (vosoritide) Lyophilized Powder for Injection

Date of Approval: November 19, 2021

Company: BioMarin Pharmaceutical Inc.

Treatment for: Achondroplasia

Voxzogo (vosoritide) is a $\mathrm{C}$ type natriuretic peptide (CNP) analog indicated to increase linear growth in pediatric patients with achondroplasia.

Fyarro (sirolimus protein-bound) Lyophilized Powder for Injectable Suspension

Date of Approval: November 22, 2021

Company: Aadi Bioscience, Inc.

Treatment for: Malignant Perivascular Epithelioid

Cell Tumor (PEComa)

Fyarro (sirolimus protein-bound particles) is an inhibitor of mechanistic target of rapamycin kinase (mTOR) for the treatment of malignant perivascular epithelioid cell tumor (PEComa).

\section{Livtencity (maribavir) Tablets}

Date of Approval: November 23, 2021

Company: Takeda Pharmaceutical Company Limited Treatment for: CMV Infection in Transplant Patients

Livtencity (maribavir) is a cytomegalovirus (CMV) pUL97 kinase inhibitor used for the treatment of post-transplant CMV infection/disease.

\section{Cytalux (pafolacianine) Injection}

Date of Approval: November 29, 2021

Company: On Target Laboratories, Inc.

Treatment for: Identification of Ovarian Cancer

During Surgery

Cytalux (pafolacianine) is a fluorescent imaging agent indicated as an adjunct for the intraoperative identification of malignant lesions in patients with ovarian cancer. 
PreHevbrio (hepatitis B vaccine (recombinant) Injection

Date of Approval: November 30, 2021

Company: VBI Vaccines Inc.

Treatment for: Hepatitis B Prevention

PreHevbrio (hepatitis B vaccine (recombinant) is a 3antigen hepatitis $B$ vaccine indicated for the prevention of hepatitis B virus (HBV) infection in adults.

\section{Lyvispah (baclofen) Oral Granules}

Date of Approval: November 22, 2021

Company: Saol Therapeutics, Inc.

Treatment for: Spasticity

Lyvispah (baclofen) is an oral granule formulation of the approved gamma-aminobutyric acid agonist baclofen indicated for the treatment of spasticity.

Reference: $w w w . d r u g s . c o m$

Information collected and compiled by

Md. Akbar Hossain

ASA University (ASAUB)

Shyamoli, Mohammadpur

Dhaka-1207, Bangladesh 